\title{
The Deceleration of Radio/Light Transmission; A Collision Mechanism Dependent Radio Signal Emission, Transmission, and Reception
}

Running title: Light/radio Collision Transmission

(C) By JING ZHANG

Lemma Technology Co.

125 N Mary Ave. 69, Sunnyvale, CA 94086 


\begin{abstract}
The fast and slow fading of radio signal transmissions or laser beam itself and its spare light confirm the presence of two kinds of collisions --- electron-photon and photon-photon collision. Photon transmissions of radio signals are either in consecutive dense photon groups in slow fading process or widespread group pattern in fast fading form. The photon transmission shapes are irregular and dynamical changes. The radio frequency shift and the wavelength reduction by the calculation demonstrate decelerations of radio/light transmission speeds due to the gradual reduced distance between two consecutive photon groups along the transmission pathway. The simultaneous presence of two radio/light transmission patterns overturns the principle of Special Relativity by Einstein. Photons from radio signals are able to affect electron movements in radio signal receiving process and electrons able to kick photons into space during radio signal emission. The radio signal and light emission and reception are due to the collision between photons and electrons. The local electromagnetic changes between atoms of antenna play the central role. Collision Mechanism explains electron-photon frequency relation of light/radio emission mechanism. Collisions between highand low-speed photons reduce the speed of high ones. The mathematical equation for the relationship between light/radio transmission speeds before and after photons collisions is established. The equation is confirmed by the calculations of well-known difference of light transmission speeds in different media. The gravities from Earth and Sun play little role during light/radio transmission.
\end{abstract}

\title{
Key words:
}

Photon and electron collisions | Collision | radio transmission | deceleration of light transmission | radio frequency shift 


\section{Introduction}

Einstein's "invariable light speed" was based solely on his visual perception without any effective physical basis. Since the human visual perception of the motion is only 40 milliseconds (ms) for continuous frame (e.g. movies using film strip) $[1,2,3,4]$, it was impossible for Einstein to detect any motion changes beyond the human limitation (e.g. changes within $10^{-9}$ second). In addition, mechanical time recording devices of a century ago were only in the sensitive range of hundred ms (the limitation of mainsprings) [5]. Therefore people in 100 years ago were unable to see or measure changes in light speed by the naked eyes or devices. Einstein's major error in 1905 was the ignorance of limitations of human eyes [1,2]. The agreement about the speed of light signal for all the observers in the relative motion is not meaningful if there is no way to tell the difference of light speed changes.

Einstein derived his formula, $\mathrm{E}=\mathrm{mc}^{2}$, based upon two postulates: "1) the laws of physics are invariant in all inertial systems; 2) the speed of light in a vacuum is the same for all observers, regardless of the motion of the light source" $[1,2]$. The second point, the speed of light, did not have an effective basis in physics, as described in the previous paragraph.

In Special Relativity (Einstein's postulation, 1905), "light in vacuum propagates with the speed $c$ (a fixed constant, independent of direction) in at least one system of inertial coordinates (the "stationary system"), regardless of the state of motion of the light source." The relative velocities are also constant [2]. Because of the constant velocity relative to the speed $\mathrm{c}$ in stationary system, Doppler Effect has Einsteinian and Newtonian Equation based on imaginary thinking, the propagation of light/radio signal as a circular shape or signal rays. The two equations separately present two different single frequency shift values from the speed $\mathrm{c}$ of the stationary system [6]. The single speed relative to speed c in stationary system logically, correctly has the single frequency shift value! However neither speed $\mathrm{c}$ of stationary system nor relative velocity has any effective measurement report. The contradiction of Doppler Effect to modern radio signal data will be showed in Method Section of this report. The acceleration of Lorentz transformation in 4-dimension spacetime in relativistic theory does not exist because no global radio frequency shift pattern (i.e. the same standard cell phone services in the world) has been found consistently to any spacetime-associated movement [9]. Therefore the radio communication data overturns the imaginary prediction of Special Relativity.

The measurement of average light speed has been attempted for many times, including $2.2 \times 10^{8} \mathrm{~m} / \mathrm{s}$ (meter/second) by Ole Rømer (1676, Denmark) [13], 3.01 x $10^{8} \mathrm{~m} / \mathrm{s}$ by James Bradley (1728, England) [14], $3.15 \times 10^{8} \mathrm{~m} / \mathrm{s}$ (laser beam) by Hippolyte Fizeau (1849, French) [15] and so on. The variation of speed values has been considered as inaccurate measurement due to Einstein's theory, invariable light speed [1, 2, 12]. However different average transmission speeds in literature before 1900 could be due to the different transmission path divided by the different time.

There are many models for radio/light transmission. Regardless of which model it is, the critical mistake is without any effective mechanism of deceleration of photon transmission in space. That is contradictory to radio signal frequency shift data. Here are the discussions for those models.

Wave model of radio/light transmission mistakes the behavior of electrons and photons purely in term of electron wave functions because of electron wave function without frequency shift properties in real photon transmission $[7,8,22,23,24]$. The electron waves for CD disk reading by Wave Model is actually from electronic oscillator because the frequency of electron waves in circuit is different from that of laser, thus it 
is not the behavior of electron and photons. It is fundamentally contradictory to the real situation of frequency shifts in photon transmission [7, 8]. There is no logic connection of the vibration rate of atoms to the light frequency in photon transmission. Therefore wave only model is completely excluded.

Particle only model for light transmission got its evidence from the diffraction of x-rays by a crystal [25]. However the approach is the same optic methods in principle as that used for particle side in particle-wave dualism model. The optic approaches have no way to observe frequency shifts in radio/light transmission, thus there is no practical application for the construction of photon transmission model. The particle-wave dualism is also impossible. The issues will be proved in Method.

Another difference between Collision Mechanism and Quantum is the issue of the source of photons. In Quantum, the emission of photon is postulated due to the release of photons from electrons during the energy level change (electron transition) [26, 27]. The hypothesis is based on the spectrum of visible light or slightly beyond the visible light range, which is generated by a tube that is filled with hydrogen gas and applied with high voltage of electricity [28, 29] (see explanation in Discussion). The absence of the measurement of the Distance between Electron and Nucleus (DEN) in the hypothesis leads to un-effective postulation and the worthless correlation of the wavelength of hydrogen light spectrum to DEN. In addition, free electron itself with high potential stage (e.g. direct voltage) in circuit board does not emit photons of radio signals or light when electric potential drops to low stage, indicating the absence of electron transition in Quantum. In this report, it is for the first time to have a mechanism with accurate frequency measurements of photons and electrons, resulting in a conclusion of that the collision of electrons with photons are the only choice for the light emission.

\section{Methods}

Solution for Wavelength Issue and the deceleration of photon transmission: Figure 1 was plotted in order to compare Einstein's water wave with the modern radio transmission signal frequency shift data [7, 8, 22]. Based on Einstein's theory, invariable light speed in a water wave transmission model is plotted on the left side. The photon wave speeds are $\mathrm{V}_{\mathrm{a}}=\mathrm{V}_{\mathrm{b}}=$ $\mathrm{V}_{\mathrm{c}}=\mathrm{V}_{\mathrm{d}}=\mathrm{V}_{\mathrm{e}}=\mathrm{V}_{\mathrm{f}}$. There are no distance changes between consecutive photon groups because of invariable light speed. Therefore there is no frequency shift, and no signal fading if Doppler Effect is not present (the issue will be discussed in later section). Theories of the model support $\mathrm{E}=\mathrm{mc}^{2}$ and Ultra-relativistic particles $\mathrm{E}^{2}=\mathrm{m}^{2} \mathrm{c}^{4}+$ $\mathrm{p}^{2} \mathrm{c}^{2}[1,2,30]$. According to radio communication data $[7,8,22]$, the continuous shift from low to high radio frequency is symbolically depicted on the right side of Figure 1. To use the frequency shift data here, it is

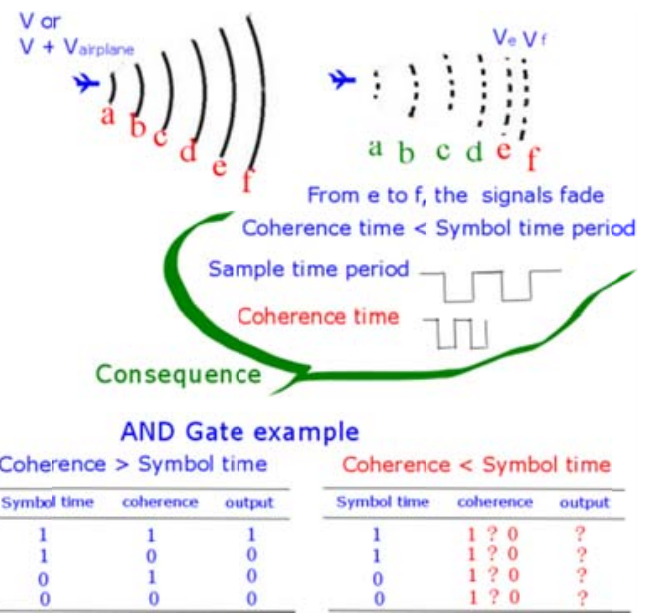

Figure 1 Legend. The Comparison of Einstein's theory with Radio Communication Data in Collision Mechanism

The picture on the left represents Einstein's theory. The radio wave on the right side is plotted 
symbolically from the radio frequency shift data [Figure 1 and 2 in ref. 7; Figure 3 and 4 in ref. 8, and 22 respectively]. The wavelength changes is verified in Calculation Section. The photon's speeds are $\mathrm{V}_{\mathrm{a}}>\ldots>\mathrm{V}_{\mathrm{e}}>\mathrm{V}_{\mathrm{f}}$. The dished line means that photons adjacent to the high-energy state photons remain intact by Collision Mechanism. The digital communication data (in Discussion) show that from a to $d$, the radio signal is normal since the coherence time $>$ symbol time period (in the early phase of the deceleration). From e to $f$, the radio signal is fading and coherence time $<$ symbol time period (in the late phase of the deceleration). Due to out of symbol time, the failure reason of the electronic receiving device is given in the table. The data confirm the result of the reduction of wavelength changes.

The presence of free photons in spaces: The density of free moving photons is present. Free moving photons are present on the surface of the earth since the sunlight is, at least, one of photon sources. Additional evidence is that a well-established example is from medical hard x-ray machines. The increase of frequency decreases the measurable intensity of x-rays [31], suggesting a limited photon output because of the exhausted photon supply in a confined electron moving space, thus supporting the presence of photon density.

No circular wave-like propagation in radio transmission and No waves with diffuse power in space (TWDP): The first evidence again the presence of "wave" in photon transmission is from solar eclipse picture (Figure 2a). Similar interactions to hydrophobic or hydrophilic forces between molecules for periodic table elements (Figure 2b) are not the cases for photons otherwise the distinguishable light rays would have not been observed in Figure 2a.
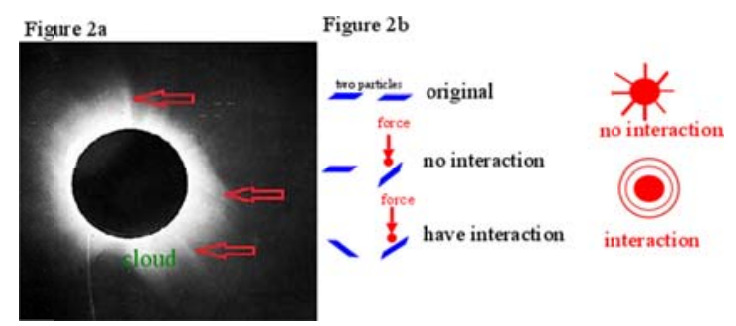

Figure 2 Legend. 2a.Solar Eclipse The solar eclipse by Eddington, S. A. (1919) was confirmed by other (https://apod.nasa.gov/apod/ap160412.html) [11]. The red arrows point to light rays. The rays are not completely straight due to the gravity effect of the moon, which is consistent with Einstein's prediction. The rays are distinguishable from its environment, indicating no interactions of the rays with surrounding photons. "Cloud" in green represent lights or spare lights between rays.

The second evidence is that the major technology in photonic methods including grating equation, the cavity resonance (or resonator), and so on is the constructive or destructive interference (Figure $3 b$ ) as its the critical data (interferogram), which is used as an evidence of "wave" [9, 10, 16, 17, 18, 19]. The interferogram are alternative color changes and were thought because of the frequency phase changes in Figure $3 \mathrm{~b}$--- "the constructive interference could shift to destructive one, and vice versa". Human eyes are not able to pick up photon group 1 and 3 or 2 and 4 as electronic device do in Figure $3 \mathrm{a}$, thus the final illusion for human eye at focus point of the intersection of two laser beams is to pick photon group 1, 2, 3, and 4. However the mirrors in entire optic systems for con/destructive interference do not have the capability dynamically to shift laser frequency phase back and forth to form alternative color changes in interferogram. Therefore the interferogram is not an effective postulation based on nothing. There is no "wave" in photon transmission (Figure 3a) [22]. The illusion like photon group 1, 2, 3, and 4 in Figure 3a can only give single solid color, but not alternative color changes due to phase shift. It is not the case of monochrome laser phase drift at the focus point of the intersection of two laser beams [20, 21]. Therefore the different frequency of control laser beam from that sample one due to the different transmission path is present to result in artificial illusion of alternative color changes as in Figure 3c. The conclusion is consistent 
to the observation of radio frequency shift data in Figure 1, indicating the presence of the deceleration of laser transmission. Because the frequency of the control laser beam is not able to match the shifted one of the sample, any calculation based in control and sample laser beam are not effective.

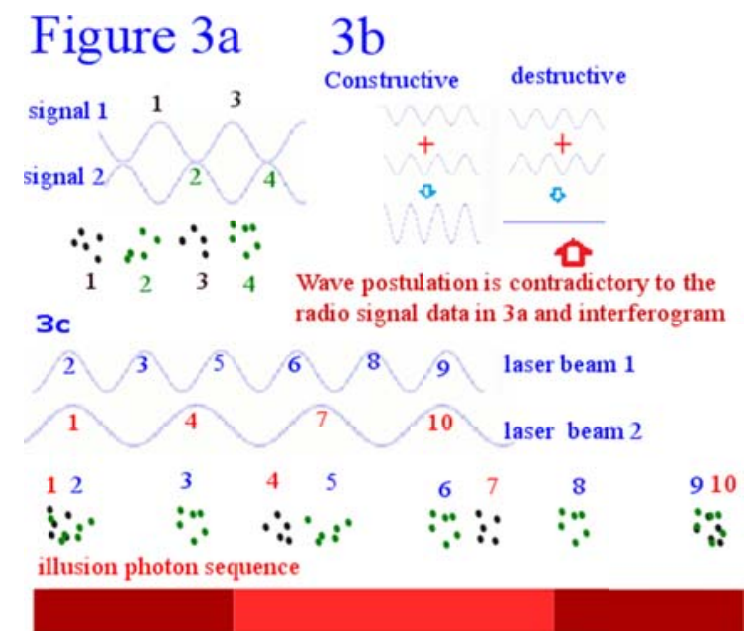

Figure 3 Legend: a. The radio signal data at left side with phase difference is symbolically depicted from data [Figure 4 in reference 22]. The corresponding photon group to each signal peak is labeled in the same number as each photon group by $1,2,3$, and 4 . It shows that photon groups from two signals do not meet one another in space, thus destructive interference is impossible at right side. $\mathbf{3 b}$. imagined wave-like laser photon transmission could have the consequence of addition or cancellation effect. The cancellation of two waves leads to destructive interference and the darkness in interferogram (actually just another different color as $3 \mathrm{c}$ in real data). 3c. Laser 1 is corresponding to green color photon groups at interval $23.4 \mathrm{~mm}$ distance, and laser 2 , to black ones at interval $39 \mathrm{~mm}$. One of every 5 green color photon groups overlaps with one of every 3 black ones. It is to show how the intersection of two different frequencies can create multiple different color illusion in interferogram.

In addition to application of interferogram, the wavelength calculation by the grating equation (light traveling distance $d\left(\sin \theta_{\mathrm{m}}\right)=\mathrm{m} \lambda, \lambda$ is wavelength) in photonic method has no physical demonstration for factor $\mathrm{m}$ to represent something equivalent to the number of wavelength in the distance $\mathrm{d}\left(\sin \theta_{\mathrm{m}}\right)$. The calculation is ineffective.

The third evidence against "wave" is from statistic result of Rician postulation --- "two waves with diffuse power (TWDP), leading to frequency shift in space" [38]. The major interference between human made radio signals has not been observed in space. Radio signals are not disturbed by one another unless frequencies are close enough to each other [41]. The disturbance happens at the physical layer of devices, but has no evidence that occurs in space. Although the devices can emit standard radio signals, signals are different from one another due to different circuit and components [45, 46,]. The bits' transmissions of radio signals in space do have occasional errors, e.g. detected by checksum, but errors with the possibility either in space or at antenna level are minor and at individual bit levels. It does not support TWDP postulation [47]. The slow fading and frequency shifts in Rician postulation are not due to TWDP. Therefore data of both photon emission and transmission in space indicate that the contribution to photon density from human radio signals is negligible, indicating additional evidence of no TWDP presence.

In summary, the imaginary con/destructive interference is not present. The multiple colors in interferogram can be explained as the result of the intersection of different frequencies of two laser beams. It proves "no destructive interference", thus indicating "no wave presence". So particles and wave dualism model is impossible. The postulations by Rician and Rayleigh as TWDP are contradictory to data of radio signals as the description above, thus suggesting that the "diffuse power" does also not exist in space $[22,38,40]$.

Dynamic changes of radio signal transmission in random pattern and Multiple frequency shifts without Doppler Effect: The statistic observations of Rician and Rayleigh fading give a clue that photon groups transmit in different paths because the intensity of signals are dynamically, randomly up and down in fading pattern (Figure 4) [38]. Rayleigh fading is within a period in microsecond range [7, 8], indicating that 
photon transmission in fast fading path only is momentarily presen [7, 8]. Both frequency shift data and statistic observation point to the same conclusion --- that the transmission path and pattern are dynamically, randomly changed with the transmission time [7, 8, 38].

Figure 4 Legend: Slow fading process is in black, fast one is in green color. Because the fading pattern is randomly up and down [38], the signal transmission distance vs its signal intensity of fast and slow fading can be depicted according to Rayleigh and Rician statistic observation to explicitly show the fading pattern.

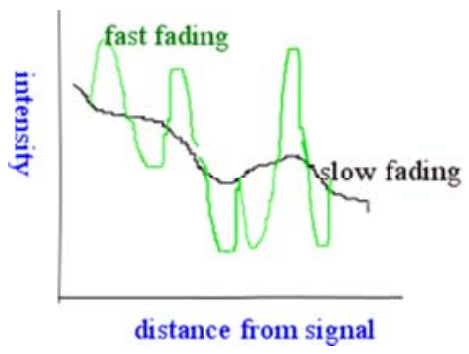

In the report by Dong et al, the experiment environment is designed according to the postulation of Doppler shift, but contradictory observation of multiple frequency shifts to the single frequency shift of Doppler Equation was the result, indicating the absence of Doppler Effect due to speed and angle [7]. The simulation by Dong et al is also meaningless since $\mathrm{Hz}$ range simulated data is different from real radio signal in $2 \mathrm{GHz}$, indicating no similarity of real signal to "simulated" data and their failure of the simulation [7]. It is contradictory to the postulation of Special Relativity (SR) that the speed relative to speed c in stationary system logically, correctly has the single illusion frequency shift [1,2]. None has ever seen the data of single frequency shift in radio signal data in reality. Although the ion beam simulation by the accelerator observed single speed change in Ion-Beam Doppler shift, ion beams are different from photon transmission (Rician and Rayleigh fading) and contradictory to the observation of continuous frequency shift photon signal data $[7,8,56]$. There is no similarity of photon transmission to ion beam transmission. The multiple frequency shift pattern of radio transmission firmly suggests to the deceleration mechanism of radio transmission along its path. Data of multiple frequency shifts are well-established in Rayleigh fading by multiple observers $[7,8]$. Accordingly the postulations of Doppler Effect and SR are completely experimentally excluded.

We do have experiences that the radio signal is stronger in certain direction than other ones. The data suggest that human perception is not able to distinguish the difference in micro- or millisecond range as that detected by the equipment $[7,8]$. In addition, sequential collisions can occur after initial collision of primary signal beam to photon (see Figure 4 and Figure 5b). The evidence to support it is the detection of signals in other directions as well. The antenna can receive signal from primary signal beam or secondary, even third sequential collision signals. The best signal direction of an antenna reflects the maximal signal photon collision with the antenna in unit time and unit area (see Figure 6). The radio signal itself has no way to gain its power in space [38]. Both slow and fast fading is due to the signal fading along its distance [38]. TWDP to explain frequency shift in Rayleigh and Rician postulation has no basis without "wave" support. So Collision Mechanism in this report is the only right answer.

\section{The evidence of photon-photon Collision in space, but not due to ray like} scattering event, no electron transition theory, and no Special Relativity:

Figure 5a demonstrates that laser beam can emit light by itself, but not from source or due to scattering, and overturns the Rayleigh hypothesis [38]. The laser source from place a and receiving at $d$ are all green color. The human observation of green color laser beam from green arrow points is not the case of "a line of sight signal between the laser source and eyes" (fits into Rayleigh's application). It does not require the scattering environment as Rayleigh postulation, indicating that photon transmission caught by human eyes is different from laser beam itself in completely different direction in space. There are two photon transmission patterns. One is laser beam and too

Figure 5a. Observation of Laser beam

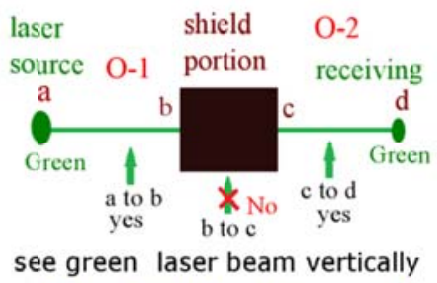

5b. Single photon is impossible and photon transmission in group

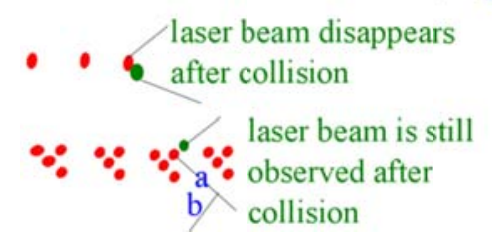


strong to make direct human eye contact. Another is spare light and weak enough for human eye to see the laser beam, indicating less dense photon group in spare light transmission than laser beam itself. The phenomena are similar to the observation of fast and slow fading in radio signal transmission (Figure 4) [38]. In the presence of free photon, significant portions of photons in laser beam sharply change transmission direction without any other physical factors in space, indicating that the only possible solution is the collision of photons from laser beam with free ones in space. That is photon-photon collision in Collision Mechanism. In addition, the sequential collision (labeled blue letter b) can occur equivalently as the initial one (labeled in blue letter a in Figure 5b). The evidence is observers of the laser beam able to see the beam from different directions (no single Rayleigh fading beam like laser). So the ray like pattern in Rayleigh fading as report by Dong et al is impossible [7].

Figure 5a indicates many photons in a group in the transmission (Figure $5 \mathrm{~b}$ ) because laser beam would have disappeared after the collision in transmission if there had been only a photon in each photon wave. It suggests that single photon emission in electron transition theory of Quantum is impossible for radio signal emission. The large scale of electron movement is only triggered by electromagnetic potential in circuits (including antenna) without the requirement of light source as the postulation of electron transition theory. So the source of photon by the hypothesis electron transition theory is excluded.

There are no two identical points with an identical radio signal intensity and frequency in its transmission pathway from radio signal transmission data (Figure 4) [7, 8, 22, 38]. So it is a wrong imaginary conclusion that "The speed of light in a vacuum is the same for all observers, regardless of the motion of the light source" in Special Relativity by Einstein [1,2]. Accordingly there is no meaningful agreement of the transmission speed or the transmission time in Quantum or Special Relativity. The dynamic continuous frequency shifts of radio signal are contradictory to the explanation of single frequency shift in Special Relativity $[1,2,7,8]$. In Figure 5a, your eyeballs capture Rayleigh fading transmission while the real light beam transmission is Rician fading. Rayleigh fading at O-1 is also different from that at O-2 according to the Rayleigh fading data of radio signals (Figure 5a) [7, 8]. In addition, it is not applicable that "laws by which the states of physical systems undergo change are not affected" in the Principle of Relativity because of the failure to find a consistent frequency shift (constant transmission speed) in radio signal data [7,8]. Therefore Special Relativity does not exist as what Einstein expects.

The deceleration of photon transmission due to the presence of photon-photon collision: The sunlight is only well-known factor that has major impact on photon density (Interference does not require frequencies close to one another). There were different radio frequency shift patterns between the sunset and night from radar data, indicating that the photons from sunlight disturb the transmission of radio signal, and suggesting the deceleration due to the presence of Photon-Photon Interactions (PPI). Since there are no waves as descriptions in particles-wave dualism (Figure 1, 2, 3 and 4) [10], PPI indicate the transmission deceleration due to the collisions between photons (no evidence suggests the influence of photon transmissions by electric charge) [22]. A similar example to radar data is that the flashlight illuminates a longer distance during the night than that in day. It is because the photon densities and levels of free photons in space are lower during night than that in daytime. The number of collisions of high-energy state photons with low ones in different densities affects the light transmission distance. Beside the fast fading radio signals occur in every consecutive photon group [Fig. 2 in ref. 7], indicating that significant number of collisions between photons occurs to allow the detection as fast fading radio signal [7]. The data is consistent to the spare light observation of laser beam in Figure 5a. Accordingly the deceleration of photon transmission in space is concluded due to significant collisions between photons in transmission path. The argument for the presence of smaller particles than photons to provide resistance and deceleration for photon transmission is negative 
because fast and slow fading data in radio signal transmission indicate the presence of two different decelerations $[38,40]$. Two decelerations are against the postulation of one deceleration media due to smaller particles than photon. It is the same argument as the different radio frequency shift pattern before and after sunset in previous example. Otherwise there is no reason to have fast and slow fading and different frequency shift after sunset in the same deceleration media. The fast and slow fading data is consistent with different radio frequency shift after sunset to make the conclusion of the deceleration of photon transmission due to Collision Mechanism.

The presence of the collision between electron and photons: The electron transition theory in quantum has no real data to support its presence as described in Introduction. The radio emission and reception are due to the collision of electron with photons. The evidence comes from the antenna in signal communication. Because the antenna in radio signal emission is not magnetic metal materials and has no closed electric circuit, the antenna does not have any major measurable magnetic field. The radio signal reception from capacitor is due to internal local electromagnetic field (EMF) changes in the antenna (Figure 6). The radio frequency in the air is similar to that in electric circuit, indicating that it is due to photon-driving-force-dependent electron movements, leading to EMF changes in antenna, thus Photon-Collision-Mediated Electron Movement (PCMEM) is suggested (Figure 6). The metal shield of point $\mathrm{B}$ in Figure 6 demonstrates that the exposure to the radio signal in the air is not required for signal transmission, but radio signals (electric current change at capacitor) indicate the presence of localized EMF changes in antenna. The requirement for metal materials of the antenna to receive radio signal and retrievable radio signals at any point (e.g. A, B, etc. in Figure 6) of antenna suggest that the local EMF changes can spread out through the alteration of local free electron movements in different directions because of the lack of major measurable magnetic field. Multiple radio frequencies can be received simultaneously in an antenna (e.g. FM/AM radio); indicating presence of multiple local EMF changes simultaneously. For radio signal emission, the transmission process is Electron-Mediated-Photon Movement (EMPM) and opposite of the receiving one. The local EMF of antenna drives the movement of electrons in metal. Therefore photon transmission paths in the space can be dynamically changed with the changes of local EMF as the observation of signal randomly, repeatedly up and down along the transmission course of the slow fading track (Figure 4). [38]. Accordingly it is evidence that photon transmission shapes are dynamically changed with the transmission times. In addition, radio signal transmissions are in groups of photons (Figure 5), suggesting that each electron kicks out many photons each time at signal emission. In this report, the best circuit to receive or emit radio signals is not the goal for the purpose.

In case of both radio emission and reception, the electronic oscillator (an electronic component) provides precise frequency of radio signal for transmitter and receiver [55]. The radio signal is amplified by different kinds of transistors to achieve different emission powers. It is not related to the postulation electron transition of atoms in antenna. The electron transition theory in quantum is contradictory to the radio technology. Thus collision of electron with photons is the only mechanism to explain electron-photon related transmission.

The radio signal emission or reception is not due to the vibration of nuclei: The spaces between any positively charged nuclei and its surrounding area have electromagnetic fields. It provides the basis for the electron movement at certain frequency (Figure 6). That is Atom-Electron-Association Environment (AEAE). A temperature change of a substance or chemical reactions can be detectable when AEAE distortions are significant enough for substances. Thermal expansion and contraction of a substance (volume changes) is the solid evidence of the disruption of original AEAE. Under the new AEAE, the distance change between nuclei leads to the electron moving frequency changes or the alteration of electron moving 
frequency results in the distance adjustment between the nuclei in solid substances.

Since the vibrations of atoms in solid substances are usually less than $10^{13}-10^{14} \mathrm{~Hz}$ and is different from visible sunlight frequency, thus photon from Sun ray are not able to shake up nuclei in metal [32, 33]. The frequencies of radio Emission or reception at antenna are not subject to the temperature changes, suggesting that vibration rates of metal atoms due to temperature changes is not the factor of radio frequency changes $[32,33]$. It is impossible to send out radio signals through nuclei vibrations.

Figure 6 Legends. Radio Signal received in Antenna. It is a well-known nature of antenna. There is no measurable EMF and no electric current flow due to absence of closed circuit on antenna. So the local EMF direction is in arbitrary direction as marked in blue arrow. The radio signal can be received at any point like A, B, C, D, and E in any kind of antenna shapes. Point $\mathrm{B}$ is connected with a capacitor to the devices with/without the metal shield in the shade area. In metal materials, the free electron movement is according to its EMF. Signal transmission by capacitor is relied on EMF change in the surrounding area of point B.

The evaluation of radio transmission: Since the light/radio transmission speed is not constant (Figure 1), the major target for the mathematical calculation is to develop the speed relationship between before and after collision. The verification of mathematic speed relationship

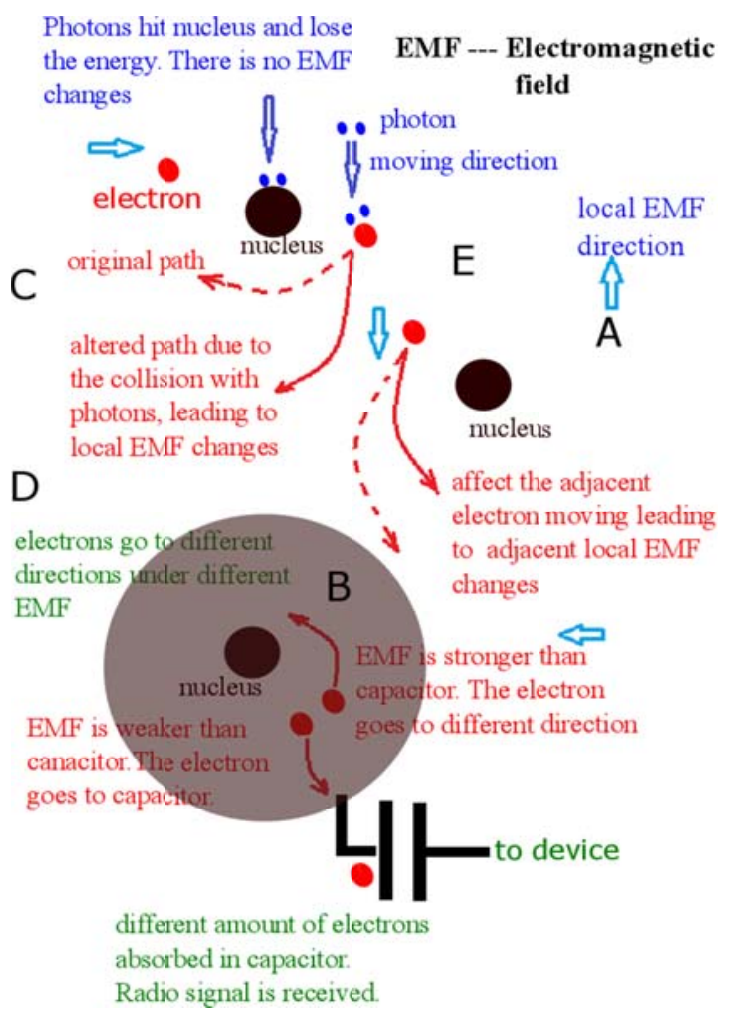
is done by using well-known different light transmission speeds in different media. The law of conservation of energy is the best choice to use for the evaluation of collisions of photons and is consistent with the observation of propagation theory $\left(\mathrm{P}_{\mathrm{fs}}=\mathrm{P}_{\mathrm{rad}} / 4 \pi \mathrm{d}^{2}\right)[34,35]$. Since the invariable light speed theory $(\mathrm{E}=$ $\mathrm{mc}^{2}$ or $\mathrm{E}^{2}=\mathrm{m}^{2} \mathrm{c}^{4}+\mathrm{p}^{2} \mathrm{c}^{2}$ ) are contradictory to the well-established observation of modern radio communication theory (Figure 1) [30], it is not appropriate to employ those equations to calculate the movements of the photons. The radio frequency shift indicates the radio speed change, suggesting that the major factor of speed is contributing to the photon energy.

\section{Results}

Electron is bigger than photon. Photons have density. There are two kinds of collisions. One is through electron-photon collision, another due to photon-photon collision. A periodic movement of an electron can hit many photons in each collision to form a photon transmission in consecutive dense photon groups, resulting in slow fading process or laser beam itself (the requirement of the line of sight signal in Rician theory). In contrast, photon-photon collisions only lead to events of two collided photon movements. Accordingly photon transmission is in widespread group pattern and a process of fast fading or spare light of laser beam. Figure 5 and the data of slow and fast fading confirm two kinds of collisions in Collision Mechanism [38]. Figure 5 indicates countless collisions leading to spare light, which is the best demonstration of "each periodic collision of an electron with many photons" because spare light does not deplete photons in laser beam (Figure 5). 
The frequency shift in Figure 1 is due to the deceleration of photon transmission. Figure 5 and 6 prove the presence of two transmission patterns of laser beam due to collisions of photons from laser beam with free moving ones and electron with photons. The influence of sunlight on radio frequency shift and shooting distance of flashlight confirm the existence of free photon. Collision Mechanism is the reason of deceleration. Figure 7 shows why there are two deceleration mechanisms separately for slow and fast fading transmission. The dense photon groups encounter relatively less collisions with free photons than ones with spread patterns.

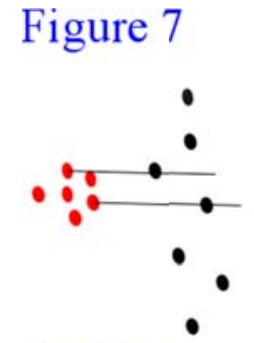

slow fading has dense photon group --high speed photons in red color --free photon in black color

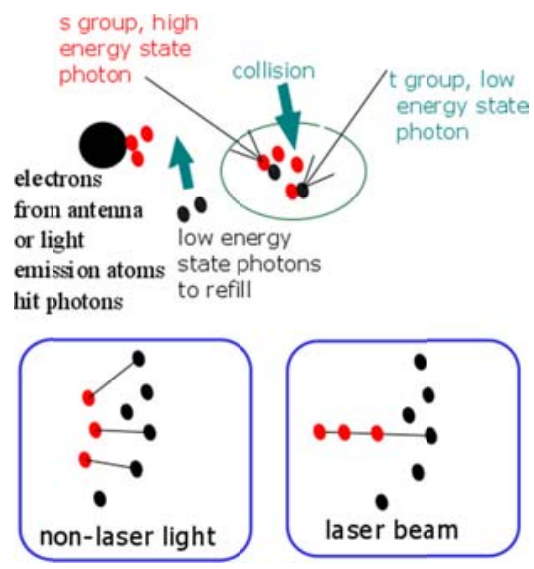

Figure 8 Legend: Collision Mechanism. Upper portion: Low energy state photons are labeled in black dots and high one in other colors. The black lines between photons represent the photon transmission path. English letters are used for groups of photons in the same way as that they are used in Calculation Section. On upper left side, electron hits photons, and those photons then collide with low energy state photons; Bottom portion: the two boxes symbolically show the difference of non-linear light and laser beam in transmission path. That is why laser beam shoots longer distance than non-linear light.

Accordingly moving particles with certain densities in the same space can randomly collide with one another. A free moving photon does not itself have any capability to accelerate since radio signal transmission is always fading, which the same as flashlight is diminishing in distance. The deceleration of photon transmission is discussed in Method. The photon transmission cannot alter its direction of movements in the space, but relies on outside force (Figure 5a). The collision of photons with others of equivalent or almost equivalent mass can lead to the dispersal of fast moving photons' speed with low ones and alter the direction of photon transmission (fast fading of radio signal and spare light in Figure 5a). Collisions of fast moving photons with molecules, atoms, or electrons result in the loss of the energy of photons in transmission direction (shield portion in Figure 5a). The changes of photon speed will be mathematically developed in Calculation. The basic requirement for Figure 8 and 9 has to be consistent with those observations of radio communication theories and Figure 5.

Figure 9 Legend: The upper left is the consequence of the collision between photons with high and low energy state. The upper right is plotted according to Figure 5a. The letter a represent the collision of photons with primary signal beam; $b$, with fast fading photon groups, or sequential collision (according Figure 5a). For the bottom one, the fast fading is according to Figure 5 and frequency shift data $[7,8]$. The intensity of slow fading are randomly up and down (Figure 4) [38]. Therefore moment 1 with high intensity is due to the collision of slow fading photon groups; 2 with weak intensity is Collision of high speed photon with low one

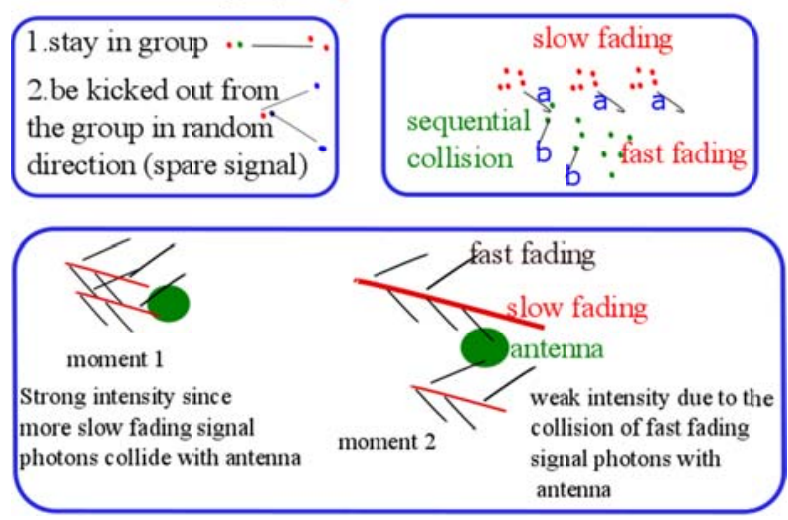
because only fast fading photon groups collide with antenna. The intensity may be increased if antenna position can be adjusted to face more photon groups of fast fading at moment 2 . Therefore the direction 
of antenna for better signal intensity is due to the amount of collisions of photon groups with antenna in unit time and unit area.

\section{Calculations}

Radio Wavelength Calculation: On the right side of Figure 1, let $\mathrm{d}_{1}=\mathrm{cd}$ (distanice between photon group c and $d$ ); $d_{2}=d e, D_{t}=$ ce (total distance); average speed $V_{\text {lav }}$ between $\mathrm{c}$ and $\mathrm{d}$ and $\mathrm{V}_{2 \mathrm{av}}, \mathrm{c}$ and $\mathrm{d} ; \mathrm{T}_{1 \mathrm{p}}$ is the period between $\mathrm{c}$ and $\mathrm{d} ; \mathrm{T}_{2 \mathrm{p}}, \mathrm{d}$ and $\mathrm{e}$. Because we know

$$
\begin{aligned}
& d_{1}+d_{2}=T_{1 p} V_{1 a v}+T_{2 p} V_{2 a v} \\
& T_{1 p}>T_{2 p}--- \text { frequency shift data }
\end{aligned}
$$

(The shift data are Figure 3 by Dorr in reference 8, Figure 4 in reference 22. Frequencies $f$ shift from low to high one; and period $\mathrm{T}$ from big to small one $\mathrm{f}=1 / \mathrm{T})$. Then we have:

$$
\begin{aligned}
& D_{t}=d_{1}+d_{2}=T_{1 p} V_{1 a v}+T_{2 p} V_{2 a v}<T_{1 p} V_{1 a v}+T_{1 p} V_{2 a v} \\
& D_{t}<T_{1 p}\left(V_{1 a v}+V_{2 a v}\right) \\
& \frac{1}{2} D_{t}<T_{1 p} \times \frac{1}{2}\left(V_{1 a v}+V_{2 a v}\right)
\end{aligned}
$$

Since $1 / 2\left(\mathrm{~V}_{1 \mathrm{av}}+\mathrm{V}_{2 \mathrm{av}}\right)$ is the average speed from photon group c to e, $\mathrm{d}_{1}=\mathrm{T}_{1 \mathrm{p}} \mathrm{x} 1 / 2\left(\mathrm{~V}_{1 \mathrm{av}}+\mathrm{V}_{2 \mathrm{av}}\right)$ and $\mathrm{d}_{1}>$ $1 / 2 D_{t}$. So it is proved that $d_{1}>d_{2}$ and the wavelength is reduced during the frequency shift process. Therefore the radio frequency pattern on the right side of Figure 1 is verified. Because of photon transmission in groups (Figure 5), it is conclusive result that the distance between two consecutive photon groups is gradually reduced along the transmission pathway (not illusion one in contrast to Special Relativity and Doppler Effect). By far it is the most effective evaluation of the wavelength of the radio/light transmission.

Einstein Equation for frequency and wavelength: Since constant light speed $\mathrm{C}=300 \mathrm{k} \mathrm{km} / \mathrm{s}$ of the laser speed is not correct by above demonstration and no effective physical basis, the result $\left(d_{1}>d_{2}\right)$ calculated by Einstein's equation wavelength $\lambda=\mathrm{C} / \mathrm{F}$ ( $\mathrm{F}$ is frequency) is not valid and contradiictory to Special Relativity. The grating equation cannot give effective wavelength measurement (see introduction section). There is only one measurable factor, frequency, out of three in the equation. Therefore the wavelength for radio/non-linear light in literature calculated by the equation $\lambda=\mathrm{C} / \mathrm{F}$ are not authentic [36]. In addition, the demonstration of $d_{1}>d_{2}$ in previous paragraph proves that there is no constant speed and wavelength in a light/radio transmission process, indicating a consistent result to coherence time change in radio signal (Figure 1). Therefore Einstein Equation is not meaningful to solve the speed-and-wavelength problem.

The evaluation of gravity effect: The reduced distance change between two consecutive photon groups is not due to gravity effect. The following evaluation can demonstrate it. It is well known that the first, second, and third cosmic velocities are $7.9,11.2$ and $16.7 \mathrm{~km} / \mathrm{s}$ respectively. The velocities give the essential speed requirements to escape the gravity of the earth and the sun. Let $\mathrm{m}=$ mass of a photon

The photon energy at third cosmic velocity: 


$$
E_{\text {photon, } 3 r d \text { cosmic } V}=\frac{1}{2} m V^{2}=\frac{1}{2} m \cdot 16.7^{2}=140 m
$$

A photon average energy at speed $=300,000 \mathrm{~km} / \mathrm{s}[15]$.

$$
E_{\text {photon }}=\frac{1}{2} m V^{2}=\frac{1}{2} m \cdot 300,000^{2}=4.5 \cdot 10^{10} m
$$

Thus in the cases of light or radio speed directions parallel to the gravitational force, the percentage of the photon energy to overcome gravity is

$$
\frac{E_{\text {photon, } 3 r d \text { cosmic } V}}{E_{\text {photon }}}=\frac{140 \mathrm{~m}}{4.5 \times 10^{10} \mathrm{~m}}=3.1 \cdot 10^{-9} \%
$$

The conclusion is too small amount of energy to contribute any effect due to the gravity in our solar system. However the majority of radio signals or laser beams are confined to the earth or the solar system, confirming previously described deceleration mechanism (not due to gravity) during photon transmission and supporting Collision Mechanism. Although the photon transmission course can be altered to very limited degree by gravity in solar eclipse (Figure 2) [11], the deceleration of photon transmission by gravity is negligible in our earth-solar system.

Collision Mechanism Calculation: According the law of the conservation of energy and based on the Collision Mechanism in Figure 8, let $\mathrm{s}=$ the number of photons in a high-energy sitate along the transmission track, $\mathrm{t}=$ the number of a low-energy state photons, and $\mathrm{m}=$ mass of a photon. Then we have the kinetic equation before and after photon collisions.

$$
\begin{aligned}
& \frac{1}{2} s m V_{1}^{2}=\frac{1}{2}(s+t) m V_{2}^{2} \\
& s V_{1}^{2}=(s+t) V_{2}^{2}----- \text { equation } 1
\end{aligned}
$$

Therefore, we have

Therefore

$$
V_{1}^{2}=V_{2}^{2} * \frac{s+t}{s}
$$

$$
V_{1}=V_{2} \sqrt{1+\frac{t}{s}}----- \text { equation } 2
$$

Equation 2 describes the speed relationship of photons before and after collision in the photon transmission course. The ratio of the collisions between the high-speed photon and low one is the only determinant factor for the speed.

From Equation 2, we can get Equation 3 as following:

$$
\frac{V_{1}}{V_{2}}=\sqrt{1+\frac{t}{s}}----- \text { equation } 3
$$

By using equation 3, we can compare the photons' speed in different media in order to confirm if Equation 2 is reasonable for the speed relation between before and after collisions. In a vacuum space, there are no 
molecules or atoms. The high-speed photon collides with low-speed one and disperses its speed during its transmission, but total energy remains essentially unchanged. However photons that collide with molecules in a molecule-containing space (e.g. air, water) are considered as a loss of energy. Let $\mathrm{x}=$ the number of photons colliding with molecules. Equation 3 in comparison of that in vacuum becomes as following equations:

$$
\frac{V_{1}}{V_{2}}=\sqrt{1+\frac{t}{s}}---- \text { in vacuum }
$$

$$
\frac{V_{1}}{V_{2 x}}=\sqrt{1+\frac{t}{s-x}}---- \text { in water }
$$

If $x>0, s-x<s$ then

$$
\sqrt{1+\frac{t}{s-x}}>\sqrt{1+\frac{t}{s}}
$$

Since $V_{1}$ is identical (the same light source) in above two equations, $V_{2}>V_{2 x}$. Therefore we proved that the speed of light in a vacuum space is faster than that in a molecule-contained space. Thus the result proves Equation 2 since it is well known that the light speed is faster in vacuum than that in water.

In case of two molecule-containing media (e.g. water and sucrose solution), $\mathrm{x}$ and $\mathrm{y}$, the above equation became as followings:

$$
\frac{V_{1}}{V_{2 x}}=\sqrt{1+\frac{t}{s-x}}---- \text { in medium } x \quad \frac{V_{1}}{V_{2 y}}=\sqrt{1+\frac{t}{s-y}}---- \text { in medium } y
$$

Where $\mathrm{x}$ and $\mathrm{y}$ separately represented the number of photons colliding with molecules (lost energy and not contribute to the light transmission). Since the densities of molecules in the media $\mathrm{x}$ and $\mathrm{y}$ were different from one another, then

$$
\sqrt{1+\frac{t}{s-x}} \neq \sqrt{1+\frac{t}{s-y}}
$$

$$
\frac{V_{1}}{V_{2 x}} \neq \frac{V_{1}}{V_{2 y}}
$$

Because of the same light source $\mathrm{V}_{1}$ for medium $\mathrm{x}$ and $\mathrm{y}, \mathrm{V}_{2 \mathrm{x}} \neq \mathrm{V}_{2 \mathrm{y}}$. Therefore the light transmits at different speeds when the light entered the medium with different molecule density or with different molecules, e.g. air, water solution and sucrose solution. It is visible for human eye if the difference of $V_{2 x}-V_{2 y}$ is big enough (see discussion section).

\section{Discussion}

The light transmission speeds in the media with different kinds of molecules were different from case to case, thus explaining our visual perception of many physical phenomena, e.g. the colorless crystal or ice in the colorless water, etc. The collisions of moving photons with molecules were nott only dependent upon the molecules' density, but also upon multiple other factors, e.g. the molecules' size, moving speed, etc. The phenomena are strong evidences supporting Collision Mechanism.

The frequency shift of radio signal and deceleration are the nature of radio/light transmission: Collision Mechanism is supported from the coherence time changes of digital communication devices (Figure 1). The coherence time $\left(\mathrm{T}_{\mathrm{c}}\right)$ is approximately inversely proportional to the maximum shifted frequency $\mathrm{f}: \mathrm{T}_{\mathrm{c}} \approx 1 / \mathrm{f}$. When the coherence time $<$ symbol time period, the digital signals fade, suggesting bandwidth changes during the radio transmission regardless the presence of motion [37]. The electronic 
device can measure the shift from $30,000,000 \mathrm{~Hz}$ to $30,000,060 \mathrm{~Hz}$ [39]. The frequency shift is present in all the radio/light transmission (e.g. 802.11, radio or television) regardless the motion [41, 42, 43]. In comparison, the small-scale changes in the frequency indicate the relatively earlier stage of the radio transmission deceleration than that observed by the radar $[22,39]$. The frequency shifts indicate the gradual decrease of the distance between two consecutive photon groups, and decisively overturns the concept that "the speed of light is invariable".

The detection of the frequency shift is usually the comparison of received signal frequency with original carrier frequency $[39,44]$. The frequency shift and signal fading are related to a number of factors like the signal emission power, distance, weather, and so on $[48,49,50]$. The frequency shift is always observed between the transmitter and receiver [51, 52, 53]. The distance of radio transmission depends on the radio emission power. The digital signal fading occurred in 20 meters for 802.11 while the radar for 1200 kilometer $[22,54]$. In addition, the more humanity is in the air, the more ineffective collision between photons and molecules. Typical examples of weather effects are the different intensities of the sunlight in cloudy raining from clear sunny day. The existence of multipath transmission events (phase changes during the transmission) indicates that both the instantaneous and constant deceleration processes is present simultaneously in single radio/light transmission pathway [7]. Therefore Collision Mechanism gives a perfect explanation for all different observations of frequency shift.

\section{The explanation of electron-photon interaction and nature physical phenomena support one another:} By Collision Mechanism, the disruptions of electron-flow mediated AEAE in the hot tungsten filament (thermal expansion) of a lamp changes electron-moving frequency to a visible light range (Figure 6), thus the collisions of electrons with surrounding photons lead light emission. The Thunderstorm is another similar case to lamp. The huge thunder proves that the events happen at the molecule level. The bigger distance between molecules in air than solid materials leads to huge thunder, but not thermal expansion. The ion beams (just like electric current) of lightning in a thunderstorm cause the electromagnetic field changes for the surrounding air molecules (AEAE disruption), leading to the electron vibration rate changes to a visible light frequency in those molecules and resulting in the lightning. The squiggly lines of lightning prove that multiple emission light sources are from surrounding air molecules, but not single light source in straight line as that of sunray. The ion beam itself does not have visible light frequency and is hazard if human has a contact with it. The 150 miles/hour wind is not able to cause any thunder or lightning because of no AEAE disruption. In the case of hydrogen tube for the development of electron transition postulation in quantum, the experimental conditions are merely a similar case to thunderstorm situation with a perfect explanation for the observation of the light spectrum of hydrogen gas tube, but not due to electron transition in Quantum [28, 29].

The metal temperature changes under the sunlight are a case of PCMEM (Figure 6). It is because photons from sunlight hit electrons and original AEAE is disrupted (see in Methods). The photon energy of man-made radio signals is weaker than that of the nature sunlight since the sunlight can increase metal temperature while radio signals has not observed, indicating the movements of electrons are strong enough to change EMF, leading to the distance change between nuclei (thermal expansion). Therefore any vibration of positive charged nuclei in antenna case is not due to photon collision, which further proves PCMEM in the antenna case (Figure 6). The stronger the sunlight is; the higher metal temperature is. Accordingly the strength of radio signals depends on how large the scale and how high the velocity of photons are. Just like the wind, the number and the speed of air molecules in the flow of the wind determines whether the wind is strong enough to wave a flag. 


\section{Conclusion}

Collision Mechanism and deceleration of photon transmission are demonstrated to be present due to electron-photon and photon-photon collision, leading to slow and fast fading process respectively. The multiple continuous frequency shifts in radio/light transmissions are against the single frequency shift model in any Doppler Effect, which is superimposed on the imagination of circular propagation of radio signal. The distance between two consecutive photon groups (not wave-like pattern) is gradually reduced along its transmission pathway, thus the deceleration of radio/light transmission is present. For light/radio transmission, Collision Mechanism is the only one to explain the deceleration of photon transmission in space and overturns Special Relativity, water wave model by Einstein, and particle-wave dualism in quantum. In light/radio emission and reception, Collision Mechanism capsizes electron transition in quantum. The mathematical evaluation provides the relationship between photon speed before and after collision and is confirmed by the well-known light speed changes in different media. Collision interactions of photons with electrons prove the radio emission and receiving mechanism. The photon-photon collisions lead the deceleration during radio/light transmission.

\section{Reference}

[1] Einstein, A. (2001) Relativity: The Special and the General Theory. Reprint of 1920 translationby Robert W. Lawson ed. (Routledge, New York), pp.48

[2] Einstein, A. (1905) Annalen der Physik 17: 891 (English translation by George Barker Jeffery and Wilfrid Perrett; 1923).

[3] Efron, R. (2001) Perception \& Psychophysics 14(3), 518-530.

[4] Davis, J. (1986) Scientific Reports 5: 7861

[5] Carle, D. D. (1969) Practical Watch Repairing, 3rd Ed. (Robert Hale Ltd, London), p91.

[6] Landau, L.D.; Lifshitz, E.M. (2005). The Classical Theory of Fields. Course of Theoretical Physics; Vol. 2. Trans. Morton Hamermech ( $4^{\text {th }}$ revised English ed). Elsevier Butterworth-Heinemann. p1-3.

[7] Dong, L; Xu, G; Ling, H. (2001) Global Telecommunication Conference, Vol. 6, p3287-3291.

[8] Dorr., B.L. (1995) Mobile Radio Technology. Vol.11, p30-60.

[9] Pospelov, M; Romalis, M. (2004) PHYSICS TODAY, July. P40.

[10] Wang, K; Xu, Q; Zhu, S.; Ma, X. S. (2019) Nature Photonics, Vol. 13, p1-7

[11] Eddington, S. A. (1919) This Month in Physics History, American Physical Society, vol.25(5), p2. 2016

[12] International Bureau of Weights and Measures, (2006) The international System of Units (SI). (Stedi Media, 75018 Paris. 8th Ed.), p112

[13] Saito, Yoshio (June 2005). AAPPS Bulletin. 15(3): 9-17.

[14] Hirshfeld, Alan (2001). Parallax: The Race to Measure the Cosmos. New York City: Henry Holt.

[15] Poincaré, H. (1904). Maxwell's Theory and Wireless Telegraphy: Part 1. New York: McGraw Publishing Co. pp. 52-55.

[16] Siegman, A. E. (2000). IEEE Journal of Selected Topics in Quantum Electronics. 6 (6): 1389-1399.

[17] Sullivan, D.B. (2001). In Lide, DR. A Century of Excellence in Measurements, Standards, and Technology. CRC Press. pp. 191-193.

[18] Evenson, K.M; et al. (1972).Physical Review Letters. 29 (19): 1346-49.

[19] White, S. E and Cataluna, M. A. (2015) Photonics. 2, 719-44

[20] Saleh, B. E. A. and Teich, M. C. (2001) Fundamentals of Photonics, (John Wiley \& Sons, Inc.).

[21] Goodman, J. W. (2000) Statistical Optics. ${ }^{\text {st }}$ ed. (Wiley-Interscience Publication) p210-221.

[22] Yau, K.S.B.; Coleman, C.J.; and Cervera, M.A. (2006) $10^{\text {th }}$. IET international Conference on 
Ionopheric Radio System and Techniques. July 18-21. p7-11.

[23] Carver Mead, (2000). Collective Electrodynamics: Quantum Foundations of Electromagnetism, MIT Press, p11, p45-46.

[24] Paul Arthur Schilpp, ed, Albert Einstein: Philosopher-Scientist, Open Court (1949), p. 51.

[25] Duane, W. (1923). The transfer in quanta of radiation momentum to matter, Proc. Natl. Acad. Sci. 9(5): $158-164$.

[26] Planck, M. (1901) Ann. Phys., 309 (3): 553-63.

[27] Kragh, H. (1999), Quantum Generations: A History of Physics in the Twentieth Century, Princeton University Press, p. 62.

[28] Lyman, T. (1906) The Astrophysical Journal, Vol. 23, No. 3, p181-210

[29] Wiese, W.L. and Fuhr, (2009) J.R J. Phys. Chem. Ref. Data. Vol. 38, No.3

[30] Dieckmann, M. E. (2005) Physical Review Letter. Vol 15, 155001.

[31] Schaaffs, W. \& Stephenson, S. T. (1957) Röntgenstrahlen/X-Rays, (Springer, Berlag oHG. Berlin), p.341

[32] Hollas, J. M. (1996) Modern Spectroscopy ( $3^{\text {rd }}$ ed.). John Wiley, p77.

[33] Banwell, C. N., McCash, E. M. (1994). Fundamentals of Molecular Spectroscopy (4 ${ }^{\text {th }}$ ed.). McGraw Hill, p71.

[34] Rosen, J. \& Gothard, L. Q. (2009) Encyclopedia of Physical Science (Infobase Publishing, New York), p155.

[35] Seybold, J. S. (2005) Introduction to RF Propagation (John Wiley and Sons, Inc., Hoboken, New Jersey), pp3-10.

[36] Halliday, D.; Resnick, R.; and Walker, J. (2011) Fundamentals of Physics, $9^{\text {th }}$ ed. (John Wiley \& Sons, Inc), p25, p417.

[37] Corrazza, G. E. (2007) Digital Satellite Communications, (Springer Science, Business Media, New York, NY, USA), p68-70.

[38] Prasad, M.S.G.; Siddaiah, P.; Redddy, L.P.; and Lekha, K. (2010) International Journal of Systems and Technologies, Vol. 3, No. 1, p139-145

[39] Roschmann, H., Theisges, W., Zoberbier, M., Brakemeier, A., Erbach, E (1994) US Patent Number $5,305,347$

[40] Abdi, A.; Tepedelenlioglu, C.; Kaveh, M.; and Giannakis, G. (2001) IEEE Communications Letters, March, p. 92 -94.

[41] Valkenburg, M.E.V. (2002) Reference for Engineers: Radio, Electronics, Computer, and Com-munications. 9th ed., (Butterworth Heinemann, Woburn, MA, USA), p35-4 to p35-13

[42] Li, C and Ng, T.S. (2003) Joint Conference on the $4^{\text {th }}$ International Conference on Information, Communications and Signal Processing and the $4^{\text {th }}$ Pacific Rim Conference on Multimedia Proceedings, Singapore, Vol.2, p.847-878.

[43] Won, K. H.; Han, J. S. and Choi, H. J. (2010) Journal of Networks, Vol.5 No. 3, p313-319

[44] Baier, A. (1991) US Patent Number 5434889

[45] Baldini, G.; Steri, G.; Giuliani, R. (2018) Encyclopedia of Information Science and Technology, $4^{\text {th }}$. edition, P. 11.

[46] Wang, W.H.; Sun, Z.; Ren, K.; Zhu, B.; Piao, S.. (2016) IEEE Transaction on Information Forensics and Security.Vol. 11(9), p2091-2106.

[47] Zhang, Q. and Xiao, J. (2017) Internation Journal of Distributed Sensor Networks. Vol.13(9), p1-7

[48] Milanovic, J.; Rimac-Drlje, S; and Majerski, I. (2010) Technical Gazette. Vol. 17, 43-52.

[49] Wehus, I.K. et al (2014) Astronomy \& Astrophysics manuscript no. PIP 104.

[50] Eyo, O.E.; Menkiti, A.I.; and Udo, S.O. (2003) Turk. J. Physics. Vol. 27, p153-160. 
[51] Vyas, A. et al. (2015) JETIR. Vol. 2 (12), p 36-40.

[52] Perisa, I., Lindner J (2005) 13th European Signal Processing Conference, (Antalya, Turkey) p1-4.

[53] Minn, H., Tarasak, P., Bhargava, V. K. (2002) Proceedings IEEE 56th Vehicular Technology Conference, (Vancouver, BC). Vol. 2, pp 1230-1234.

[54] Stein, J .C., (1998) Intersil Corporation. Vol 2401, 1998.

[55] Bottom, V.E. (Retrieved on 2012) Proceedings of the 35th Annual Frequency Control Symposium 1981. Ieee-uffc.org.

[56] Hasselkamp, D., Mondry, E.; Scharmann (1979) Zeitschrift fur Physik A. 289(2), 151-155. 